\title{
Two New Flavan-Flavanones from Sarcandra hainanensis
}

\author{
Cong-Mei CAO, ${ }^{a, b}$ Li-Jia Xu, ${ }^{a, b}$ Yong Peng, ${ }^{a, b}$ Qing-Wen ShI, ${ }^{c}$ and Pei-Gen XIAO ${ }^{*, a, b}$ \\ ${ }^{a}$ Institute of Medicinal Plant Development, Chinese Academy of Medical Science, Peking Union Medical College; Beijing \\ 100193, P. R. China: ${ }^{b}$ Key Laboratory of Bioactive Substances and Resources Utilization of Chinese Herbal Medicine \\ (Peking Union Medical College), Ministry of Education; Beijing 100193, P.R. China: and ${ }^{c}$ Laboratory of Medicinal \\ Natural Product Chemistry, School of Pharmaceutical Sciences, Hebei Medical University; 361 Zhongshan East Road, \\ 050017 Shijiazhuang, Hebei Province, P. R. China.
}

Received May 4, 2010; accepted July 23, 2010; published online July 29, 2010

\begin{abstract}
Chemical study of the whole plants of Sarcandra hainanensis yielded two new biflavonoids with a flavan-flavanone skeleton, sarcandrone C (1), D (2) and 6 known compounds (3-8). Structures were elucidated on the basis of NMR spectroscopic methods.
\end{abstract}

Key words biflavonoid; flavan-flavanone; Sarcandra hainanensis; Chloranthaceae; NMR

The genus Sarcandra (family Chloranthaceae) of three species mainly grows in South and Southeast Asia. Two species of them, S. glabra (THUNB) NAKAI and $S$. hainanensis (PEI) Swamy et BAILEY, are distributed in south of China. ${ }^{1)} S$. hainanensis is an endemic shrub or tree to China. It was used to invigorate blood circulation and eliminate blood-stasis in folk medicine. ${ }^{2}$ Previous chemical studies of Sarcandra species have led to the isolation of chalcones and sesquiterpenes. ${ }^{3-6)}$ As a continuous study on the chemical constituents of $S$. hainanensis, ${ }^{6,7)}$ two new flavan-flavanones (1, 2, Fig. 1) together with 6 known compounds $(\mathbf{3}-\mathbf{8})$ were isolated.

\section{Results and Discussion}

Compound 1 was obtained as colorless powder. The negative ion high resolution-electrospray ionization (HR-ESI)MS of 1 rise a $[\mathrm{M}-\mathrm{H}]^{-}$peak at $m / z 553.1859$ corresponding to $\mathrm{C}_{33} \mathrm{H}_{30} \mathrm{O}_{8}$. In according with the molecular formula, 33 carbon signals in the ${ }^{13} \mathrm{C}$-NMR spectrum of $\mathbf{1}$ were categorized by distortionless enhancement by polarization transfer (DEPT) experiments as one carbonyl, twelve $s p^{2}$ methines, twelve $s p^{2}$ quaternary, three methoxyls, two $s p^{3}$ methylenes and three $s p^{3}$ methines carbons. Apart from the three methoxyls, the remaining 30 carbons comprising its scaffold as two $\mathrm{C}_{6}-\mathrm{C}_{3}-\mathrm{C}_{6}$ units with four benzene rings suggested that compound $\mathbf{1}$ was a flavanoid dimer.

The characteristic signals of a flavan moiety as a $\mathrm{AB}_{2} \mathrm{C}$

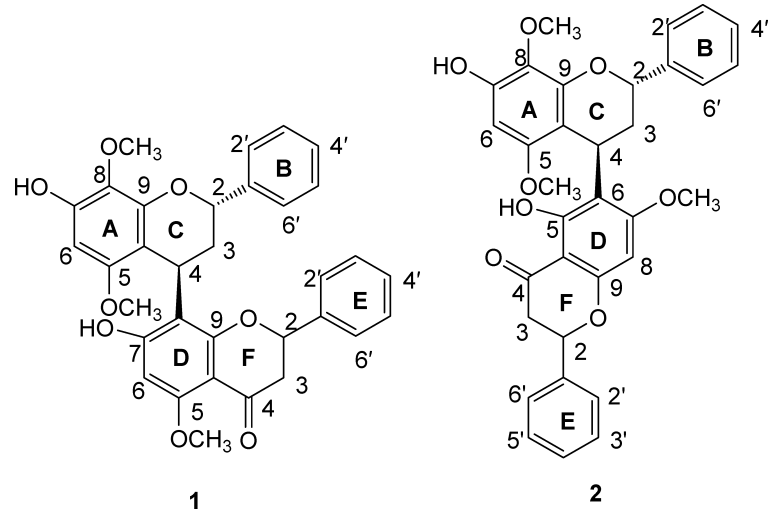

Fig. 1. Structures of Compounds $\mathbf{1}$ and $\mathbf{2}$ coupling system at $\delta_{\mathrm{H}} 5.09,2.32,2.07$ and 4.67 were observed in the ${ }^{1} \mathrm{H}-\mathrm{NMR}$ spectrum of $\mathbf{1}$ (Table 1). Another spin system at $\delta_{\mathrm{H}} 5.49$ and 2.91 suggested a flavanone terminal unit. This flavanone was further supported by the signal at $\delta_{\mathrm{C}}$ 189.7 (C-4, F) of 1 (Table 1). From the data discussed above, 1 was considered to be a flavan moiety joined to a flavanone nucleus.

The positions of the interflavan linkages were deduced from the heteronuclear multiple bond connectivity (HMBC) experiment. HMBC correlation peaks of the signal at $\delta_{\mathrm{H}} 4.67$ $(\mathrm{H}-4, \mathrm{C})$ with $\delta_{\mathrm{C}} 162.4(\mathrm{C}-7, \mathrm{D}), 109.9(\mathrm{C}-8, \mathrm{D})$ and 161.5 $(\mathrm{C}-9, \mathrm{D})$ combined with the HMBC correlation at $\delta_{\mathrm{H}} 2.07$ $(\mathrm{H}-3 \mathrm{a}, \mathrm{C})$ with $\delta_{\mathrm{C}} 109.9(\mathrm{C}-8, \mathrm{D})$ suggested a C-4 (C)-C-8 (D) linkage between the units of flavan and flavanone of $\mathbf{1}$.

In the ${ }^{1} \mathrm{H}-\mathrm{NMR}$ spectrum of $\mathbf{1}$, a multiplet at $\delta_{\mathrm{H}} 7.41-$ $7.30(10 \mathrm{H}, \mathrm{m})$ and two singlets at $\delta_{\mathrm{H}} 6.20(1 \mathrm{H}, \mathrm{s}), 6.03(1 \mathrm{H}$, s) appeared in aromatic region, these information indicated that $\mathbf{1}$ has two pentasubstituted and two monosubstituted benzene rings (Table 1). The HMBC experiment of 1 confirmed and extended these deductions. The HMBC correlations of the signal at $\delta_{\mathrm{H}} 6.20(\mathrm{C}-6, \mathrm{~A})$ with the signals at $\delta_{\mathrm{C}} 154.7$ (C-5, A), 149.8 (C-7, A), 129.3 (C-8, A) and 100.8 (C-10, $\mathrm{A} / \mathrm{C}$ ) illustrated this proton was connected to C-6 (A) (Fig. 2). While the HMBC correlations of the signal at $\delta_{\mathrm{H}} 6.03(\mathrm{C}-$ 6, D) to the signals at $\delta_{\mathrm{C}} 160.5(\mathrm{C}-5, \mathrm{D}), 162.4(\mathrm{C}-7, \mathrm{D})$, $109.9(\mathrm{C}-8, \mathrm{D})$ and $106.3(\mathrm{C}-10, \mathrm{D} / \mathrm{F})$ implied this unsubstituted proton of ring D was at C-6 (D) (Fig. 2). Additionally, the ${ }^{1} \mathrm{H}-\mathrm{NMR}$ spectrum of $\mathbf{1}$ exhibited resonances of three methoxyl singlets at $\delta_{\mathrm{H}} 3.87(3 \mathrm{H}, \mathrm{s}), 3.86(3 \mathrm{H}, \mathrm{s})$ and 3.64 $(3 \mathrm{H}, \mathrm{s})$. The HMBC correlations of these three singlets confirmed the location of the methoxyl groups as C-5 (D), C-8 (A) and C-5 (A), respectively. These deductions were further verified by the nuclear Overhauser effects (NOEs) between 5- $\mathrm{OCH}_{3}$ (D) and H-6 (D) together with the NOEs between 5$\mathrm{OCH}_{3}$ (A) and H-6 (A) (Fig. 3).

The relative stereochemistry of compound 1 could be deduced by the coupling constants of the spin systems in its ${ }^{1} \mathrm{H}$ NMR spectrum and the NOEs in its nuclear Overhauser effect spectroscopy (NOESY) spectrum. $J_{2,3 \mathrm{a}(\mathrm{C})}=11.0 \mathrm{~Hz}$ and $J_{2,3 \mathrm{e}(\mathrm{C})}=2.0 \mathrm{~Hz}$ indicated $\mathrm{H}-2$ retain as an axial bond. The equatorial orientation of $\mathrm{H}-4$ was confirmed by its coupling constant of $J_{4,3 \mathrm{a}(\mathrm{C})}=5.5 \mathrm{~Hz}$ and $J_{4,3 \mathrm{e}(\mathrm{C})}=2.0 \mathrm{~Hz} .{ }^{8,9)}$ Both H-3a (C) and $\mathrm{H}-3 \mathrm{e}$ (C) showed connectivity to $\mathrm{H}-2$ (C) and $\mathrm{H}-4$ 
Table 1. ${ }^{1} \mathrm{H}$ - and ${ }^{13} \mathrm{C}-\mathrm{NMR}$ Data of Compounds $\mathbf{1}$ and $\mathbf{2}$ in $\mathrm{CDCl}_{3}\left(500 \mathrm{MHz}\right.$ for ${ }^{1} \mathrm{H}$ and $125 \mathrm{MHz}$ for $\left.{ }^{13} \mathrm{C}\right)$

\begin{tabular}{|c|c|c|c|c|c|}
\hline \multicolumn{2}{|c|}{ Position } & \multicolumn{2}{|r|}{1} & \multicolumn{2}{|r|}{2} \\
\hline Ring & Carbon & $\delta_{\mathrm{C}}$ & $\delta_{\mathrm{H}}(J$ in $\mathrm{Hz})$ & $\delta_{\mathrm{C}}$ & $\delta_{\mathrm{H}}(J$ in $\mathrm{Hz})$ \\
\hline \multirow[t]{10}{*}{$\mathrm{A} / \mathrm{C}$} & 2 & 75.8 & $5.09, \mathrm{dd}(11.0,2.0)$ & 75.8 & $5.08, \mathrm{dd}(11.0,2.0)$ \\
\hline & 3 & 37.3 & $2.32, \mathrm{dt}(14.0,2.0)$ & 37.5 & $2.32, \mathrm{dt}(13.5,2.0)$ \\
\hline & & & 2.07, ddd $(14.0,11.0,5.5)$ & & 2.07, ddd $(13.5,11.0,6.0)$ \\
\hline & 4 & 27.4 & $4.67, \mathrm{dd}(5.5,2.0)$ & 27.4 & 4.72, dd $(6.0,2.0)$ \\
\hline & 5 & 154.7 & & 154.7 & \\
\hline & 6 & 91.3 & $6.20, \mathrm{~s}$ & 91.3 & $6.21, \mathrm{~s}$ \\
\hline & 7 & 149.8 & & 149.8 & \\
\hline & 8 & 129.3 & & 129.3 & \\
\hline & 9 & 148.9 & & 149.0 & \\
\hline & 10 & 100.8 & & 100.8 & \\
\hline \multirow[t]{4}{*}{$\mathrm{B}$} & $1^{\prime}$ & 140.8 & & 140.7 & \\
\hline & $2^{\prime}, 6^{\prime}$ & 126.1 & $7.41-7.30, \mathrm{~m}$ & 126.1 & $7.41-7.30, \mathrm{~m}$ \\
\hline & $3^{\prime}, 5^{\prime}$ & 128.5 & & 128.5 & \\
\hline & $4^{\prime}$ & 128.0 & & 128.0 & \\
\hline \multirow[t]{9}{*}{$\mathrm{D} / \mathrm{F}$} & 2 & 79.1 & $5.49, \mathrm{dd}(13.0,3.5)$ & 78.6 & $5.46, \mathrm{dd}(12.0,3.0)$ \\
\hline & 3 & 45.9 & $2.91, \mathrm{~m}$ & 45.1 & $3.01, \mathrm{~m}$ \\
\hline & $4(\mathrm{C}=\mathrm{O})$ & 189.7 & & 189.5 & \\
\hline & 5 & 160.5 & & 162.2 & \\
\hline & 6 & 94.5 & $6.03, \mathrm{~s}$ & 109.9 & \\
\hline & 7 & 162.4 & & 160.5 & \\
\hline & 8 & 109.9 & & 94.4 & $6.01, \mathrm{~s}$ \\
\hline & 9 & 161.5 & & 161.2 & \\
\hline & 10 & 106.3 & & 106.4 & \\
\hline \multirow[t]{4}{*}{$\mathrm{E}$} & $1^{\prime}$ & 139.2 & & 138.9 & \\
\hline & $2^{\prime}, 6^{\prime}$ & 125.5 & $7.41-7.30, \mathrm{~m}$ & 125.6 & $7.41-7.30, \mathrm{~m}$ \\
\hline & $3^{\prime}, 5^{\prime}$ & 128.7 & & 128.7 & \\
\hline & $4^{\prime}$ & 128.3 & & 128.3 & \\
\hline \multirow[t]{4}{*}{ OMe } & $5(\mathrm{~A})-\mathrm{OCH}_{3}$ & 56.1 & $3.64, \mathrm{~s}$ & 56.1 & $3.69, \mathrm{~s}$ \\
\hline & $8(\mathrm{~A})-\mathrm{OCH}_{3}$ & 61.4 & $3.86, \mathrm{~s}$ & 61.4 & $3.86, \mathrm{~s}$ \\
\hline & $5(\mathrm{D})-\mathrm{OCH}_{3}$ & 56.0 & $3.87, \mathrm{~s}$ & & \\
\hline & $7(\mathrm{D})-\mathrm{OCH}_{3}$ & & & 56.0 & $3.87, \mathrm{~s}$ \\
\hline
\end{tabular}

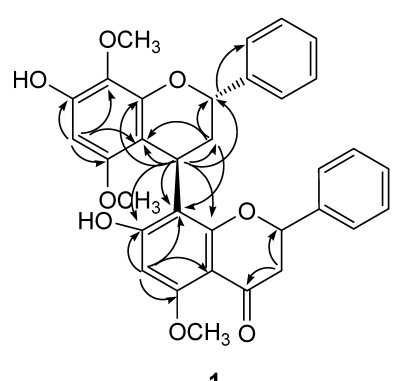

1

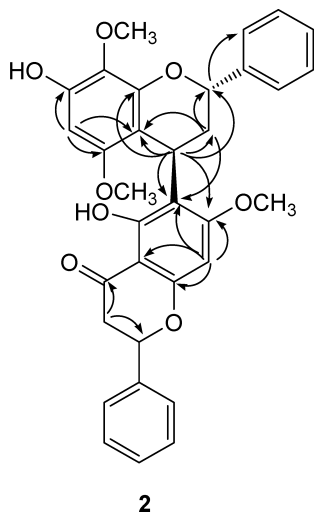

Fig. 2. Key HMBC Correlations $(\mathrm{H} \rightarrow \mathrm{C})$ of $\mathbf{1}$ and $\mathbf{2}$

(C) in ${ }^{1} \mathrm{H}-{ }^{1} \mathrm{H}$ correlation spectroscopy (COSY) spectrum of compound 1. However, no NOE was observed between $\mathrm{H}-3 \mathrm{a}$ (C) and $\mathrm{H}-4$ (C) while $\mathrm{H}-3 \mathrm{e}(\mathrm{C})$ exhibited NOEs to both $\mathrm{H}-2$ and H-4 (Fig. 3). These results were in agreement with their relative stereochemistry. A $S$ absolute configuration of compound 1 at $\mathrm{C}-4$ (ring $\mathrm{C}$ ) was determined by a negative Cotton effect of $\lambda_{\max }(\Delta \varepsilon) 214.5\left(-2.192 \times 10^{4}\right) \mathrm{nm}$ and a positive Cotton effect of $\lambda_{\max }(\Delta \varepsilon) 209.0\left(2.037 \times 10^{4}\right) \mathrm{nm} .{ }^{10)}$ Therefore, it was presumed that compound 1 process a $2 S, 4 S(\mathrm{C}$ ring) absolute configuration. Therefore, the structure of compound 1 was established as $(2 S, 4 S)$-7-hydroxy-5,8-dimethoxyflavan $(4 \rightarrow 8)$-7-hydroxy-5-methoxy-flavanone and it was
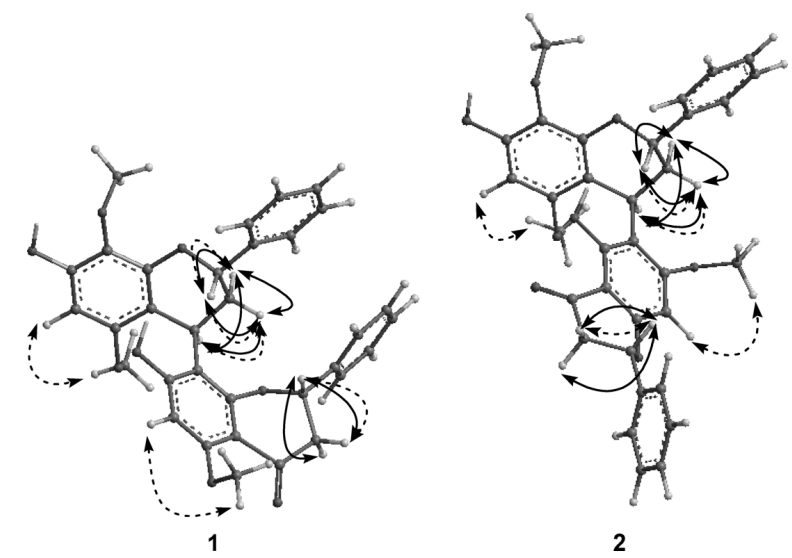

Fig. 3. Calculated Conformation by MM2 for Compounds $\mathbf{1}$ and $\mathbf{2}$

named as sarcandrone $\mathrm{C}$ (Fig. 1).

Compounds 2 possessed a molecular formula of $\mathrm{C}_{33} \mathrm{H}_{30} \mathrm{O}_{8}$ as derived from HR-ESI-MS at $\mathrm{m} / z 553.1859\left([\mathrm{M}-\mathrm{H}]^{-}\right)$, which was exactly the same as compound $\mathbf{1}$. With the structure of $\mathbf{1}$ in hand, the structure of compound $\mathbf{2}$ was easily deduced by its HMBC and NOESY data as well as comparison of the NMR data of $\mathbf{1}$ and $\mathbf{2}$.

The ${ }^{1} \mathrm{H}$ - and ${ }^{13} \mathrm{C}-\mathrm{NMR}$ data of $\mathbf{2}$ resembled those of $\mathbf{1}$, suggesting a similar structural relationship. The ${ }^{13} \mathrm{C}-\mathrm{NMR}$ spectrum of $\mathbf{2}$ differed from that of $\mathbf{1}$ by some shifted carbon signals. The deshielding shifted carbon signals at $\delta_{\mathrm{C}} 45.1,78.6$, 
94.4, 138.9, 161.2 and 189.5 were attributed to $\mathrm{C}-3$ (F), C-2 (F), C-8 (D), C-1' (E), C-9 (D/F) and C-4 (F), on the other hand, shielding shifted carbon signals at $\delta 37.5$ and 106.4 were assigned to $\mathrm{C}-3(\mathrm{C})$ and $\mathrm{C}-10(\mathrm{D} / \mathrm{F})$, respectively (Table 1). The distinctions mentioned above suggested that compound 2 differed from 1 by its interflavan linkage as C-4 (C)-C-6 (D) and the substitutions of ring D. The position of linkage in the flavanone moiety of ring D at C-6 was confirmed by the HMBC correlations of $\delta_{\mathrm{H}} 6.01(\mathrm{H}-8, \mathrm{D})$ to $\delta_{\mathrm{C}}$ 109.9 (C-6, D), 160.5 (C-7, D), 161.2 (C-9, D/F) and 106.4 (C-10, D/F) (Fig. 2). It is worthy to be mentioned that the location of the methoxyl group at C-7 (D) could be further defined by the NOESY spectrum of 2, which showed correlation between $\delta_{\mathrm{H}} 6.01(\mathrm{H}-8, \mathrm{D})$ and 3.87 (OMe-7, D) (Fig. 3).

The coupling constant of $\mathrm{AB}_{2} \mathrm{C}$ spin systems in ring $\mathrm{C}$ $\left[J_{2,3 \mathrm{a}(\mathrm{C})}=11.0 \mathrm{~Hz} ; J_{3 \mathrm{a}, 4(\mathrm{C})}=6.0 \mathrm{~Hz}\right]$ of 2 was similar to that of 1. It suggested that $\mathbf{2}$ had an identical relative configuration as $\mathbf{1}$ had. The NOEs in the NOESY spectrum of $\mathbf{2}$ could confirm its relative configuration. ${ }^{8,9)}$ Different stereochemistry of 2 from that of 1 mainly reflected on the NOEs of H-2 (C) and $\mathrm{H}-4$ (C). These two protons of 2 displayed weak NOEs to H3 e but no NOEs to H-3a. It might be caused by the weakness of the signals or difference of the dihedral angle between $\mathrm{H}-2$ (C) and H-3a (C) (Fig. 3). And it could be deduced 2 also had a $2 S, 4 S$ (ring $\mathrm{C}$ ) absolute configuration from its Cotton effects $\left[\lambda_{\max }(\Delta \varepsilon) 216.5\left(-3.140 \times 10^{4}\right), 209.0\left(2.730 \times 10^{4}\right)\right.$ $\mathrm{nm}] .{ }^{10)}$ Herein, the structure of compound 2 could be defined as $(2 S, 4 S)$-7-hydroxy-5,8-dimethoxyflavan-(4-6)-5-hydroxy-7-methoxy-flavanone and it was named as sarcandrone D (Fig. 1).

By comparison with the NMR data reported in literatures, ${ }^{11-13)}$ the 6 known compounds were identified as 7-hydroxy-5,6-dimethoxyflavanone (3), 7-hydroxy-5-methoxyflavanone (4), naringenin-4',7-dimethyl ether (5), kaempferol (6), 3,4',5,7-tetrahydroxyflavanone-3-O-glucoside (7) and kaempferol 3-O-glucoside (8).

\section{Experimental}

General Experimental Procedures UV spectra were measured on a Shimadzu UV-2550 UV-visible spectrophotometer. Optical rotations were made on a Perkin-Elmer 341 polarimeter at room temperature. CD spectra were measured on a JASCO J-725 spectropolarimeter in $\mathrm{MeOH}$. IR spectra were measured on a NEXUS-470 FTIR spectrophotometer. ${ }^{1} \mathrm{H}-(500 \mathrm{MHz})$ and ${ }^{13} \mathrm{C}-(125 \mathrm{MHz}) \mathrm{NMR}$ spectra were measured on a Bruker Avance DRX500 spectrometer. Chemical shifts were referenced to the residual solvent signal $\left(\mathrm{CDCl}_{3}: \delta_{\mathrm{H}} 7.26, \delta_{\mathrm{C}} 77.0\right)$. HR-ESI-MS spectra were performed on APEX Q-FT-MS/MS spectrometer. All solvents used were of analytical grade (Beijing Chemical Plant, Beijing, People's Republic of China). Silica gel (300 - 400 mesh, Qingdao Marine Chemical Plant, Qingdao, People's Republic of China), $C_{18}$ reversed-phase silica gel (150-200 mesh, Merck), Sephadex LH-20 gel (pharmacia) and MCI gel (CHP20P, 75-150 $\mu$, Mitsubishi Chemical Industries Ltd.) were used for column chromatography, and precoated silica gel $\mathrm{GF}_{254}$ plates (Qingdao Marine Chemical Plant) were used for TLC. Chem 3D ultra 8.0 was used for calculating MM2.

Plant Material The whole plants of Sarcandra hainanensis were collected in Baishan couty, Hainan province, China, in April 2007 and were authenticated by Professor Pei-Gen Xiao of Institute of Medicinal Plant Development, Chinese Academy of Medicinal Science. A voucher specimen (SH070706) has been deposited in the Herbarium of Institute of Medicinal Plant Development, Chinese Academy of Medical Science and Peking Union Medical College.

Extraction and Isolation The air dried, chipped whole plants of $S$ hainanensis $(29 \mathrm{~kg})$ were exhaustively extracted with $95 \%$ ethanol under reflux for twice $(2101 \times 2)$. The extracts were evaporated to yield $2928 \mathrm{~g}$ of green residue. The EtOH extract was suspended in water saturated with
$\mathrm{NaCl}$ and then extracted with petroleum ether, $\mathrm{CH}_{2} \mathrm{Cl}_{2}$, EtOAc and $n$-BuOH in order.

The $\mathrm{CH}_{2} \mathrm{Cl}_{2}$-soluble portion was evaporated to leave a residue $(720.3 \mathrm{~g})$. A portion $(611.0 \mathrm{~g})$ of it was subjected to CC of silica gel (200-300 mesh, 3000 g). Five fractions ( $\mathrm{C} 1$ to $\mathrm{C} 5$ ) were collected according to TLC. Fr. C3 was further chromatographed on silica gel column with a gradient of $\mathrm{CH}_{2} \mathrm{Cl}_{2}-\mathrm{EtOAc}$ with increasing polarity to give 15 subfractions (C3-1 to $\mathrm{C} 3-$ 15). Fr. C3-9 (8.0 g) was subjected to a CC (silica gel 200-300 mesh, $250 \mathrm{~g})$ eluting with petroleum ether-EtOAc $(5: 2-1: 2)$ to give 8 subfractions (C3-9-1 to C3-9-8). C3-9-3 was subjected to a column of Sephadex LH-20 eluting with $\mathrm{CHCl}_{3}-\mathrm{MeOH}(2: 3)$ to get compounds $3(5.6 \mathrm{mg})$ and 4 (2.0 mg). C3-9-6 was chromatographed over a column chromatography (silica gel 200-300 mesh, $170 \mathrm{~g}$ ) eluting with petroleum $\mathrm{CHCl}_{3}-\mathrm{MeOH}$ $(1: 0-10: 1)$ to give 4 subfractions (C3-9-6-1 to C3-9-6-4). C3-9-6-2 was further chromatographed on silica gel column with a gradient of EtOAc-MeOH $(1: 0-30: 1)$ to get 3 subfractions. Purification of C3-9-6-22 on a Sephadex LH 20 eluted with $\mathrm{CHCl}_{3}-\mathrm{MeOH}(2: 3)$ yeilded compound $5(5.2 \mathrm{mg})$. C3-9-7 was chromatographed over a dry column chromatography (silica gel 200-300 mesh, 250 g) eluting with petroleum ether-EtOAc (2:1) to give 3 subfractions (C3-9-7-1 to C3-9-7-3). Fr. C3-9-7-2 was purified by a column of Sephadex LH 20 eluted with $\mathrm{CHCl}_{3}-\mathrm{MeOH}(2: 3)$ and $\mathrm{MeOH}-\mathrm{H}_{2} \mathrm{O}(7: 3)$ repeatedly to afford compounds $\mathbf{1}(10.3 \mathrm{mg})$ and 2 (3.5 mg).

The EtOAc-soluble portion was separated into neutral (En) and acidic (Ea) parts by extracting with $\mathrm{NaHCO}_{3}$ aqueous solution and acidifying with $\mathrm{HCl}$. Fr. Ea was subjected to a ODS column eluting with $\mathrm{MeOH}-\mathrm{H}_{2} \mathrm{O}$ $(35: 65-100: 0)$ to afford Ea 1 -Ea 8 . Ea 5 was chromatograpied by a column of Sephadex LH $20\left(\mathrm{MeOH}: \mathrm{H}_{2} \mathrm{O} 7: 3\right)$. Eleven subfractions were collected basing on TLC. Ea 5-11 was further purified by Sephadex LH 20 chromatography to afford compound $6(10.4 \mathrm{mg})$. Fr. En $(17.6 \mathrm{~g})$ was subjected to a MCI HP20 column chromatography and 9 subfractions (En 1En 9) were collected. En 2 (2.6 g) was submitted to a column of silica gel and eluted with $\mathrm{CHCl}_{3}-\mathrm{MeOH}-\mathrm{H}_{2} \mathrm{O}(20: 1: 0.1-1: 1: 0.1)$ to furnish En 21 -En 2-16. En 2-16 was chromatographed on a column of Sephadex LH 20 eluting with $\mathrm{CHCl}_{3}-\mathrm{MeOH}(2: 3)$ to get En 2-16-1-En 2-16-5. En 2-16-2 and En 2-16-4 were purified by Sephadex LH 20 column chromatography $\left(\mathrm{CHCl}_{3}-\mathrm{MeOH} 2: 3\right.$ and $\left.\mathrm{MeOH}-\mathrm{H}_{2} \mathrm{O} 6: 4\right)$ to yield compound $7(43.6 \mathrm{mg})$ and $8(8.9 \mathrm{mg})$, respectively.

Sarcandrone C (1): Colorless powder $(\mathrm{MeOH}) ;[\alpha]_{\mathrm{D}}^{20}+46(c=8.60 \times$ $\left.10^{-3}, \mathrm{MeOH}\right) ; \mathrm{UV}(\mathrm{MeOH}) \lambda_{\max }(\log \varepsilon) 241$ (3.73), 289 (3.63) nm; CD

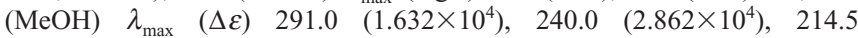
$\left(-2.192 \times 10^{4}\right), 209.0\left(2.037 \times 10^{4}\right)$; IR $(\mathrm{KBr}) v_{\max }\left(\mathrm{cm}^{-1}\right) 3392,2920,2842$, $1647,1597,1497,1462,1448,1412,1285,1107,1030,698 ;{ }^{1} \mathrm{H}-$ and ${ }^{13} \mathrm{C}-$ NMR data see Table 1; HR-ESI-MS m/z 553.1859 (Calcd for $\mathrm{C}_{33} \mathrm{H}_{30} \mathrm{O}_{8}$ 553.1868, $\left.[\mathrm{M}-\mathrm{H}]^{-}\right)$.

Sarcandrone D (2): Colorless powder $(\mathrm{MeOH}) ;[\alpha]_{\mathrm{D}}^{20}-37(c=8.00 \times$ $\left.10^{-3}, \mathrm{MeOH}\right) ; \mathrm{UV}(\mathrm{MeOH}) \lambda_{\max }(\log \varepsilon) 240(4.15), 289$ (4.02) nm; CD $(\mathrm{MeOH}) \quad \lambda_{\max }(\Delta \varepsilon) 286.5\left(2.190 \times 10^{4}\right), \quad 240.5 \quad\left(-2.034 \times 10^{4}\right), \quad 216.5$ $\left(-3.140 \times 10^{4}\right), 209.0\left(2.730 \times 10^{4}\right)$; IR $(\mathrm{KBr}) v_{\max }\left(\mathrm{cm}^{-1}\right) 3421,2956,2852$, $1641,1596,1498,1463,1448,1412,1285,1107,1030,698 ;{ }^{1} \mathrm{H}-$ and ${ }^{13} \mathrm{C}-$ NMR data see Table 1; HR-ESI-MS m/z 553.1859 (Calcd for $\mathrm{C}_{33} \mathrm{H}_{30} \mathrm{O}_{8}$ $\left.553.1868,[\mathrm{M}-\mathrm{H}]^{-}\right)$.

Acknowledgment This project was financially supported by the National Natural Science Foundation of China, Grant (No. 30530860). We are grateful to Kui Chen, Hainan Branch Institute of Medicinal Plant, Chinese Academy of Medical Science, for the collection of plant materials.

\section{References}

1) Cheng Y. Q., Chen D. Z., Wu G. F., Cheng P. S., Zhu P. Z., "Flora of China," Vol. 20, Science Press, Beijing, 1982, p. 77.

2) Xiao P. G., "Modern Chinese Materia Medica," Vol. 3, Chemical Industry Press, Beijing, 2002, pp. 195-199.

3) Tsui W. Y., Brown G. D., Phytochemistry, 43, 819-821 (1996).

4) Li Y., Zhang D. M., Li J. B., Yu S. S., Li Y., Luo Y. M., J. Nat. Prod., 69, 616-620 (2006)

5) Cao C. M., Peng Y., Shi Q. W., Xiao P. G., Chemistry \& Biodiversity, 5, 219-238 (2008)

6) Cao C. M., Xu L. J., Chen K., Peng Y., Xiao P. G., China J. Chin. Mater. Med., 34, 1009-1010 (2009).

7) Cao C. M., Peng Y., Xu L. J., Yang J. S., Wang Y. J., Xiao P. G., Chem. Pharm. Bull., 57, 743-746 (2009).

8) Birch A. J., Dahl C. J., Pelter A., Tetrahedron Lett., 8, 481-487 (1967). 
9) Ogundaini A., Farah M., Perera P., Samuelsson G., Bohlin L., J. Nat. Prod., 59, 587—590 (1996).

10) Barrett M. W., Klyne W., Scopes P. M., Fletcher A. C., Porter L. J., Haslam E., J. Chem. Soc., Perkin Trans. 1, 1979, 2375-2377 (1979).

11) Luo Y. M., Liu A. H., Yu B. W., Kang L. J., Huang L. Q., Chin. Phar- maceut. J., 40, 1296-1298 (2005).

12) Kamperdick C., Van N. H., Sung T. V., Phytochemistry, 61, 991—994 (2002).

13) Yu D. Q., Yang J. S. H., "Fen Xi Hua Xue Shou Ce," Vol. 7, Chemical Industry Press, Beijing, 1999, pp. 301, 316, 820, 837, 838. 